\title{
Optimized speed profile design of a vehicle platoon considering road inclinations
}

\author{
Balázs Németh and Péter Gáspár \\ Systems and Control Laboratory, \\ Computer and Automation Research Institute, \\ Hungarian Academy of Sciences, Hungary \\ Tel: +361-2796171; Fax: +361-4667503; e-mail: \{bnemeth;gaspar\}@sztaki.hu
}

\begin{abstract}
The paper focuses on the design of a platoon control system which takes into consideration safe travel by using the string stability theorem and the knowledge of the inclinations of the road along the route of the platoon. By choosing the speed of the platoon fitting in with the inclinations of the road the number of unnecessary accelerations and brakings can be reduced, thus so can the operations of the actuators of the vehicles, i.e. the driveline and the brake system. Although the longitudinal dynamics of the vehicles is formulated in a linear control-oriented model, the non-linear performance of the road inclinations and safety requirements based on the string stability are taken into consideration. The design of the platoon control is based on the robust $\mathcal{H}_{\infty}$ control theory.
\end{abstract}

Keywords - cruise control, platoon control, $\mathcal{H}_{\infty}$ control, robust stability and performance, optimization, vehicle control.

\section{Introduction and motivation}

In the paper a method in which saving energy and fuel consumption are taken into consideration is developed for platoon systems. The term platoon is used to describe several vehicles operated under automatic control as a unit when they are traveling at the same speed with relatively small inter-vehicle spacings. Platoon operations may have advantages in terms of increasing highway capacity and decreasing fuel consumption.

The thoughts of platoon control were motivated by intelligent highway systems and road infrastructure, see the PATH program in California and the MOC-ITS program in Japan. PATH "Partners for Advanced Transportation Technology" involves vehicle-highway cooperation and communication, and "science of driving" investigations on driving behavior. In terms of platoon control the concept of string stability to a class of interconnected systems is generalized and sufficient conditions to guarantee their string stability are sought, see $[14,24,26]$. MOC/ITS "Development of Energy-saving ITS Technology" is aimed at saving energy and reducing $\mathrm{CO}_{2}$ emission in road traffic and includes $R \& D$ of automated heavy truck platoons. In the project the between vehicles in the platoon is reduced, which will save energy and reduce $\mathrm{CO} 2$ emission 
$[8,10]$. The European programmes were based on the existing road networks and infrastructure and focused mainly on commercial vehicles with their existing sensors and actuators, see [4, 13, 25]. The main goal of the projects was to examine the operation of platoons on public motorways with full interaction with other vehicles. The safety and stability of each vehicle in the platoon is guaranteed by string stability, see e.g. [23, 26].

Several models and control strategies have been published in the topic of fuel-optimal intelligent transportation systems. [20] proposes a simplified powertrain model for intelligent vehicle applications. The model is tested using field measurements and is demonstrated to produce vehicle power, fuel consumption, acceleration, speed, and position estimates. Paper [5] classifies several modeling approaches for vehicle fuel consumption and emission, such as microscopic, mesoscopic and macroscopic modeling methods. A road type and congestion level estimation method combined with the principal components analysis is presented in [30]. This method is used for a variety of purposes, e.g. traffic information systems, intelligent real-time vehicle control systems, and energy consumption/emissions estimation. [2] proposes a modal-emissions modeling approach based on physical phenomena associated with vehicle operation and emissions. An eco-cruise control system, in which the road topographical data is involved to optimize fuel consumption, is presented in [22].

In the Hungarian TruckDAS project an automated vehicle platoon of heavy vehicles was developed. The goal of the project was to analyze the control algorithms and synthesize the experimental results, see [27]. Several questions which were related to junctions, coupling, driver behavior, technical failures, etc., had to be answered, see [21]. Within the framework of the present project we are developing a system specifically designed for commercial vehicle platforms, where traditional positioning methods (e.g. GPS) are managed together with other data (e.g. speed, lateral acceleration, radar) supplied by sensors on the vehicle. Vehicles travel in a column by using a distributed control method. In such a system, amplitudes of different types and sizes may occur due to the uncertainties of positioning and communication. These are primarily longitudinal, but some road sections may create lateral amplitudes. In addition to the lower comfort level, these amplitudes create risks in traffic safety. The preliminary results of speed profile optimization are found in [18].

In the paper a control design method which calculates optimal longitudinal forces using the road inclinations is proposed. In the control design method additional knowledge such as road inclinations and speed limits are taken into consideration. The method is based on the assumption that information about the road is available. By choosing the appropriate speed the number of unnecessary accelerations and brakings and their durations can be significantly reduced. Consequently, energy required by the actuators and fuel consumption can be reduced.

For the better understanding of the control idea, a few typical traffic situations are presented. In the first example there is a downhill slope, where the speed of the vehicle increases. If the driving force is reduced in advance, the speed of the vehicle decreases before entering the slope, thus the speed increases from a lower value and reaches the speed limit later. Consequently, the brake system can be activated later or it may not be necessary to activate it at all. In the second example there is a speed limit on the road, which imposes different strategies, e.g., rapid speed reduction with sudden braking, slow speed reduction on a longer road. In the second solution the adhesion coefficient of the road can be exploited therefore the activation of the brake can be reduced. Similar results can be presented when the speed limit has been lifted. 
Several methods in which the road conditions are taken into consideration have already been proposed, see $[11,17,19]$. The look-ahead control methods assume that information about the future disturbances to the controlled system is available. To find a compromise solution between fuel consumption and travelling time leads to an optimization problem. The optimization was handled by using various methods. In [9] the approach was evaluated in real experiments. This idea can be extended to a platoon system, since the method can be applied in the leader vehicle. Here for safety reasons the interaction between the members must be considered and the string stability of the platoon must be guaranteed. In practice the prediction about the slope of the road can be obtained in two ways: either a contour map which contains the level lines is used, or an estimation method is applied. In the former case a map used in other navigation tasks can be extended with slope information, which is applied next time along the same route. Several methods have been proposed for slope estimation. They use cameras, laser/inertial profilometers, differential GPS or a GPS/INS systems, see $[1,12,7]$.

The purpose of the paper is to extend the string stable platoon control with the road conditions. Using the different performance specifications a control-oriented model of a platoon system is formulated. Uncertainties of the model, which are caused by neglected components, and unknown parameters are also modeled as unstructured dynamics. The controller of the platoon system is designed by using a robust $\mathcal{H}_{\infty}$ method, which guarantees both disturbance attenuation and robustness against uncertainties. The schematic structure of the controlled platoon system is shown in Figure 1. A platoon control requires various measured signals, such as position, speed

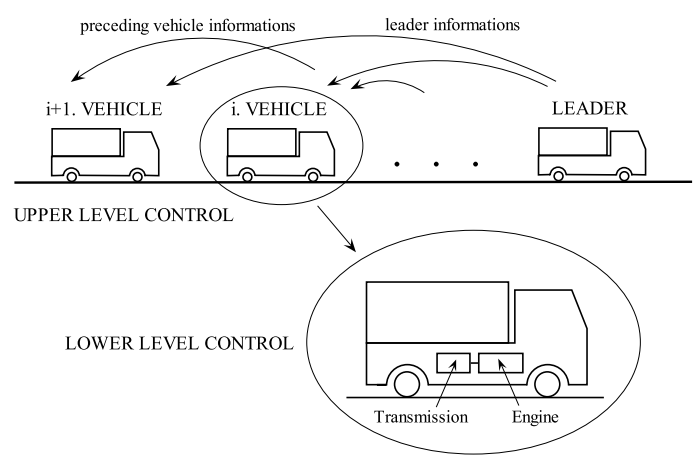

Figure 1: Structure of a platoon system

and acceleration of the leader and the preceding vehicles. Thus, the platoon control requires that information about the position, speed and acceleration of a vehicle are transmitted to the following vehicle in order to ensure string stability. Besides these signals the road conditions are assumed to be available in the vehicles (road slope, speed limit). Since the safe and economical movement of the platoon is determined by the leader vehicle, it is crucial that the leader vehicle use the road conditions. From these signals the control system of each vehicle calculates its optimal longitudinal control force.

This paper is organized as follows: Section 2 formulates the road conditions and presents the optimization of vehicle cruise control by the appropriate choice of prediction weights. Section 3 extends the optimality results to a platoon system. Section 4 presents the model based $\mathcal{H}_{\infty}$ longitudinal control design. Section 5 shows the operation of the platoon control on a transportational route with real data. 


\section{Consideration of road inclinations in cruise control}

\subsection{Weighting factors according to road inclinations}

The relationship between the optimal speed and road inclinations was a topic of an earlier paper. Thus, the thoughts are only briefly summarized. The results in a detailed form are found in [18]. The route of the vehicle can be divided into $n$ sections using $n+1$ number of points as Figure 2 shows. The sections of the route are not necessarily of equal lengths $s_{i}$. The rates of the inclinations of the road and those of the speed limits are assumed to be known at the endpoints of each section. The acceleration of the vehicle is considered to be constant between section points.

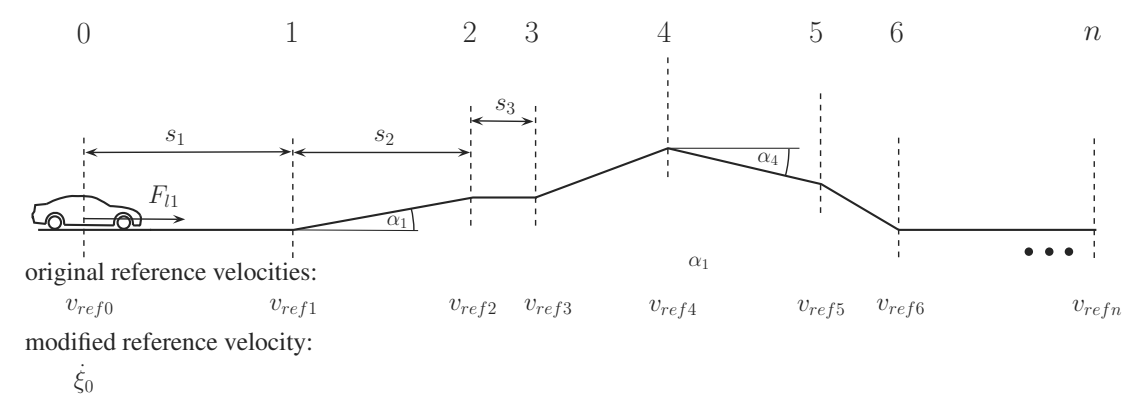

Figure 2: Division of the road

The speeds of the vehicle are described at each section point of the road. The speed at section point $j$ should reach a predefined reference speed $v_{r e f, j}^{2} j \in[1, n]$, which is usually the maximum speed of the vehicle (speed limit). It is an important goal to track the momentary value of the speed, which is formulated in the following form: $\dot{\xi}_{0}^{2} \rightarrow v_{r e f, 0}^{2}$. A weight $Q$ is applied to the momentary (initial) speed and weights $\gamma_{1}, \gamma_{2}, \ldots, \gamma_{n}$ are applied to the reference speeds. The weights should sum up to one, i.e., $\gamma_{1}+\gamma_{2}+\ldots+\gamma_{n}+Q=1$. While the weights $\gamma_{i}$ represent the rate of the road conditions, weight $Q$ determines the tracking requirement of the momentary reference speed $v_{r e f, 0}$. By making an appropriate selection of the prediction weights $Q, \gamma_{i}$ the importance of the road condition is considered. The optimal selection of weights has an important role, i.e. to achieve a balance between the momentary speed and the effect of the road slope, i.e., a balance between speed and the economy parameters.

A control-oriented vehicle model in which reference speeds and prediction weights are taken into consideration is constructed. The speed of a vehicle is calculated:

$$
\dot{\xi}_{0}=\sqrt{\vartheta-2 s_{1}(1-Q)\left(\ddot{\xi}_{0}+g \sin \alpha_{1}\right)}
$$

where $\ddot{\xi}_{0}$ represents the actual longitudinal acceleration, $s_{1}$ and $\alpha_{1}$ are the length and road inclination angle of the forthcoming section, $g$ is the acceleration of gravity. Value $\vartheta$ depends on the road slopes, the reference speeds and the prediction weights:

$$
\vartheta=Q v_{r e f, 0}^{2}+\sum_{i=1}^{n} \gamma_{i} v_{r e f, i}^{2}+\frac{2}{m}(1-Q) \sum_{i=1}^{n} s_{i} F_{d i, r} \sum_{j=i}^{n} \gamma_{j}
$$

where $F_{d i, r}$ is force resistance from road inclination on the $i^{t h}$ road section. Consequently, the road conditions can be considered by speed tracking. The calculation of $\lambda$ requires the measurement of longitudinal acceleration $\ddot{\xi}_{0}$. 


\subsection{Optimization of the vehicle cruise control}

In this subsection the task is to find an optimal selection of the prediction weights in such a way that both the minimization of control force and the traveling time are taken into consideration. The vehicle cruise control problem can be divided into two optimization problems in the following forms:

- Optimization 1: The longitudinal control force must be minimized, i.e., $\left|F_{l 1}\right| \rightarrow$ Min. Instead, in practice the $F_{l 1}^{2} \rightarrow M i n$ ! optimization is used because of the simpler numerical computation. The advantage of $F_{l 1}$ minimization is the consideration of driving and braking maneuvers simultaneously.

- Optimization 2: The difference between momentary speed and reference speed must be minimized, i.e., $\left|v_{r e f 0}-\dot{\xi}_{0}\right| \rightarrow \operatorname{Min}$.

The two optimization criteria lead to different optimal solutions. In the first criterion the road inclinations and speed limits are taken into consideration by using appropriately chosen weights $\hat{Q}, \hat{\gamma}_{i}$. At the same time the second criterion is optimal if the predicted information is neglected. In the latter case the prediction weights are noted by $\breve{Q}, \breve{\gamma}_{i}$.

The first criterion is met by the formulation of a quadratic optimization problem. It leads to the following form:

$$
\hat{F}_{l 1}^{2}\left(\hat{Q}, \hat{\gamma}_{i}\right)=\left(\beta_{0}(\hat{Q})+\beta_{1}(\hat{Q}) \hat{\gamma}_{1}+\beta_{2}(\hat{Q}) \hat{\gamma}_{2}+\ldots+\beta_{n}(\hat{Q}) \hat{\gamma}_{n}\right)^{2}
$$

This task is nonlinear because of the prediction weights. In the first optimization the prediction weight $\hat{Q}$ is fixed, and this fixed $\hat{Q}$ is modified from 0 to 1 . The optimization task is formulated in the following matrix form: Since the optimization task is linear in the sense of prediction weights $\hat{\gamma}_{i},(3)$ is formulated in the following matrix form:

$$
\hat{F}_{l 1}^{2}(\Gamma)=\frac{1}{2} \Gamma^{T} \Phi \Gamma-\kappa \Gamma
$$

where the matrix $\Gamma$ is $\Gamma=\left[\begin{array}{lllll}\hat{\gamma}_{1} & \cdots & \hat{\gamma}_{i} & \cdots & \hat{\gamma}_{n}\end{array}\right]^{T}$ and the matrix $\Phi$ comes from the rearrangement of (3). Thus, the problem leads to a quadratic programming task. In this paper the trust-regionreflective algorithm is used, which exhibits strong convergence properties and global and quadratic convergence $[3,6]$. Trust-region-reflective algorithm is the subspace trust-region method based on the interior-reflective Newton method. Each iteration involves the approximate solution of a large linear system using the method of preconditioned conjugate gradients.

The condition analysis is crucial, since it is related to the appropriation of the numerical solution. E.g. with a flat road and constant speed limits the values of $\beta_{i}(\hat{Q})(1 \leq i \leq n)$ are approximately the same. Since in this case the elements of matrix $\Phi$ are equal to each other, the matrix $\Phi$ is singular. Consequently, the computation of $\Phi^{-1}$ is difficult or impossible, and the condition number of $\Phi$ is very high. Since in practice several similar situations can be obtained, a numerical algorithm should be applied which is able to handle the poor conditioning system. The Levenberg-Marquardt algorithm used for this purpose, see [16]. In this method the original matrix $\Phi$ is increased by an identity matrix $I$ multiplied by a small number $(\delta>0): \hat{\Phi}=\Phi+\delta I$. By the Levenberg-Marquardt algorithm the condition number of $\Phi$ can be reduced significantly, which 
helps solve the optimization task. In the next step the quadratic optimization task is derived: $\Gamma=-\hat{\Phi}^{-1} \kappa$.

The second criterion is also taken into consideration. The optimal solution can be determined in a relatively easy way since the vehicle tracks the predefined speed if the road conditions are not considered. Consequently, the optimal solution is achieved by selecting the weights in the following way: $\breve{Q}=1$ and $\breve{\gamma}_{i}=0, i \in[1, n]$.

Finally, a balance between the two performances must be achieved, which is based on a tuning of the designed prediction weights. The first criterion is met by selecting prediction weights $\hat{Q}$, $\hat{\gamma}_{i}$. The second performance is met by selecting constant prediction weights $\breve{Q}=1, \breve{\gamma}_{i}=0$. In the proposed method two further performance weights, i.e., $R_{1}$ and $R_{2}$, are introduced. Performance weight $R_{1}\left(0 \leq R_{1} \leq 1\right)$ is related to the importance of the minimization of the longitudinal control force, while performance weight $R_{2}\left(0 \leq R_{2} \leq 1\right)$ is related to the minimization of traveling time. There is a constraint according to the performance weights $R_{1}+R_{2}=1$. Thus the performance weights, which guarantee balance between optimizations tasks, are calculated in the following expressions:

$$
\begin{aligned}
& \bar{Q}=R_{1} \hat{Q}+R_{2} \breve{Q}=R_{1} \hat{Q}+R_{2} \\
& \bar{\gamma}_{i}=R_{1} \hat{\gamma}_{i}+R_{2} \breve{\gamma}_{i}=R_{1} \hat{\gamma}_{i}, \quad i \in\{1, n\}
\end{aligned}
$$

Note that in this method it is necessary to normalize the values of $\hat{Q}, \hat{\gamma}_{i}$ because of the comparability to $\breve{Q}, \breve{\gamma}_{i}$. Based on the calculated performance weights the reference speed can be determined by using (1). In practice the numerical computations result in optimal prediction weights, which may change very sharply as a jump signal. In order to avoid this phenomenon the prediction weights are filtered by low-pass filters to obtain smooth signals.

\section{Design of the leader speed based on an optimization method}

In this section the method will be extended to vehicles in a platoon. The main idea behind the design is that each vehicle in the platoon is able to calculate its speed independently of the other vehicles. Since traveling in a platoon requires the same speed, the optimal speed must be modified according to the other vehicles. In the platoon, the speed of the leader vehicle determines the speed of all the vehicles. The goal is to determine the common speed at which the speeds of the members are as close as possible to their own optimal speed.

In the first step the optimal prediction weights are set $\left\{\bar{Q}_{j} ; \bar{\gamma}_{i, j}\right\}, i \in[1 ; n]$ where $n$ is the number of division points. Then the modified reference speeds of all the vehicles $\lambda_{j}, j \in[1 ; m]$ where $m$ is the number of the vehicles in the platoon are calculated. During the calculation the road inclinations and the speed limits are taken into consideration, however, the interaction between the vehicles is not considered. In the case of a platoon each vehicle has its own optimal reference speed $\lambda_{j}$. Moreover, speeds of the vehicles are not independent of each other, because the speed of the leader $\lambda_{1}$ influences the speed of every member of platoon $\dot{\xi}_{0, j}$. The goal is to find an optimal reference speed for the leader $\bar{\lambda}_{1}$.

Before the required speed of the leader vehicle $\bar{\lambda}_{1}$ is designed a second - intermediate - step is needed. It is important to note that there is an interaction between the speeds of the vehicles in a 
platoon. If a preceding vehicle changes its speed, the follower vehicles will modify their speeds and track the motion of the preceding vehicle within a short time. The members of the platoon are not independent of the leader, therefore it is necessary to formulate the relationship between the speed of the $j^{\text {th }}$ platoon member $\dot{\xi}_{0, j}$ and the leader and the preceding vehicles. It is formulated with a transfer function with its input and output. The output $Y_{j}=\mathcal{F}\left[\begin{array}{lll}\mathcal{L}\left(\ddot{\xi}_{0, j}\right) & \mathcal{L}\left(\dot{\xi}_{0, j}\right) \quad \mathcal{L}\left(\xi_{0, j}\right)\end{array}\right]^{T}$ contains the information, i.e., the position, speed and acceleration of the vehicle, which is sent to the follower vehicles. The input $U_{j}=\mathcal{G}\left[\begin{array}{lllll}\mathcal{L}\left(\ddot{\xi}_{0, j-1}\right) & \mathcal{L}\left(\dot{\xi}_{0, j-1}\right) & \mathcal{L}\left(\xi_{0, j-1}\right) & \mathcal{L}\left(\dot{\xi}_{0}\right) & \mathcal{L}\left(\xi_{0}\right)\end{array}\right]^{T}$ contains the position, speed, the acceleration of the preceding vehicle and the leader. The transfer function between $U_{j}$ and $Y_{j}$ is $G_{j, c l}=K_{j} G_{j} /\left(1+K_{j} G_{j}\right)$ with the controller of the $j^{\text {th }}$ vehicle $K_{j}$ and its longitudinal dynamics $G_{j}$. Similarly, the effect of the leader and the preceding vehicles on the $(j+1)^{t h}$ platoon member is formulated: $Y_{j+1}=G_{j+1, c l} U_{j+1}$, where $U_{j+1}=\left[\begin{array}{cc}G_{j, c l} \\ 0 & I\end{array}\right] U_{j}$, which finally leads to

$$
Y_{j+1}=G_{j+1, c l}\left[\begin{array}{cc}
G_{j, c l} \\
0 & I
\end{array}\right] U_{j}
$$

Consequently, the speed of the $j^{\text {th }}$ vehicle is determined by the next formula:

$$
\mathcal{L}\left[\dot{\xi}_{0, j}\right]=\left[\begin{array}{l}
0 \\
1 \\
0
\end{array}\right]^{T} Y_{j}=\left[\begin{array}{l}
0 \\
1 \\
0
\end{array}\right]^{T} G_{j, c l} \prod_{k=2}^{j-1}\left[\begin{array}{cr}
G_{k, c l} \\
0 & I
\end{array}\right] G_{1, c l} \bar{\lambda}_{1}=\hat{G}_{j} \bar{\lambda}_{1}
$$

The value of $\hat{G}_{j}$ is used for the computation of the optimal reference speed of the platoon $\bar{\lambda}_{1}$.

In the third step the required reference speed of the leader vehicle $\bar{\lambda}_{1}$ is designed. The aim of the design is that the generated speeds of all the vehicles $\dot{\xi}_{0, j}$ are as close to as their modified reference speed $\lambda_{j}$ as possible:

$$
\sum_{j=1}^{m}\left|\lambda_{j}-\dot{\xi}_{0, j}\right| \rightarrow \text { Min }
$$

Since the speed of the $j^{\text {th }}$ vehicle is formulated as $\dot{\xi}_{0, j}=\hat{G}_{j} \bar{\lambda}_{1}$, the following optimization form is used:

$$
\sum_{j=1}^{m}\left(\lambda_{j}-\hat{G}_{j} \bar{\lambda}_{1}\right)^{2}=\sum_{j=1}^{m} \lambda_{j}^{2}+\sum_{j=1}^{m}\left(\hat{G}_{j} \bar{\lambda}_{1}\right)^{2}-2 \sum_{j=1}^{m}\left(\lambda_{j} \hat{G}_{j} \bar{\lambda}_{1}\right) \rightarrow 0
$$

where $\hat{G}_{j}=\prod_{k=1}^{j-1} \hat{G}_{k}$. It can be stated that in (9) the only unknown variable is $\bar{\lambda}_{1}$. The optimization leads to the following equation:

$$
\bar{\lambda}_{1} \sum_{j=1}^{m} \hat{G}_{j}^{2}-\sum_{j=1}^{m}\left(\lambda_{j} \hat{G}_{j}\right)=0 .
$$

The solution of the optimization problem can be achieved. The deduction of the optimization of $\bar{\lambda}_{1}$ is as follows:

$$
\bar{\lambda}_{1}=\frac{\sum_{j=1}^{m}\left(\lambda_{j} \prod_{k=1}^{j-1} \hat{G}_{k}\right)}{\sum_{j=1}^{m}\left(\prod_{k=1}^{j-1} \hat{G}_{k}\right)^{2}}
$$




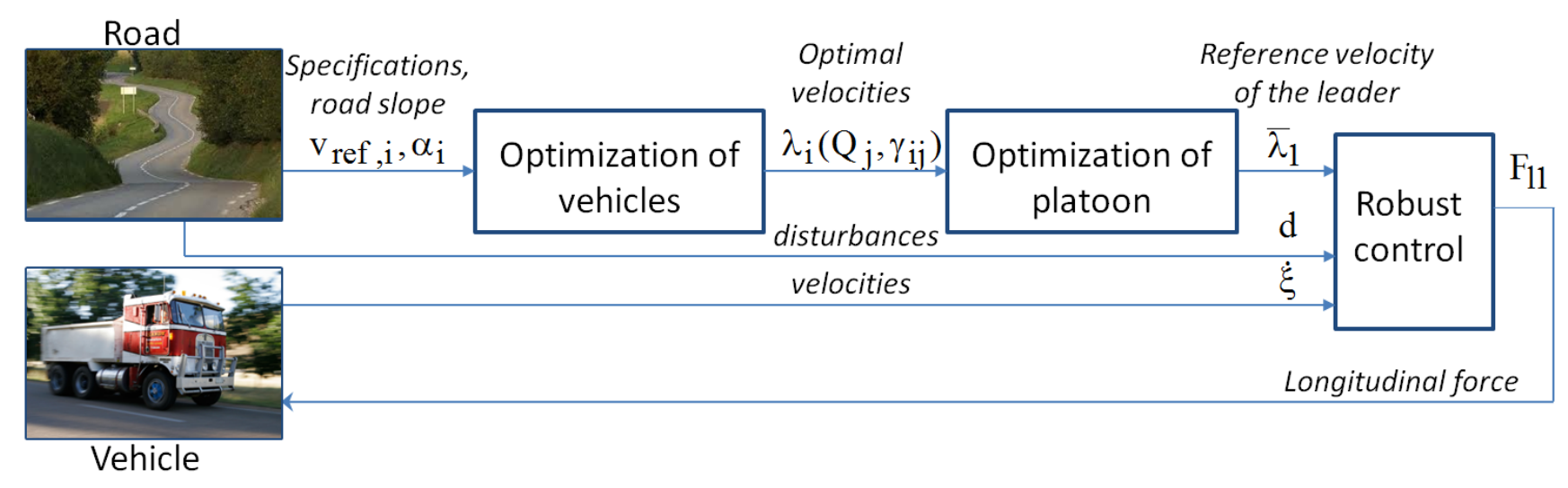

Figure 3: Architecture of the control system

It means that the leader vehicle must track the required reference speed $\bar{\lambda}_{1}$.

The calculation of the longitudinal force, which is illustrated in Figure 3, is performed in three steps:

- The optimization of speeds of the vehicles is based on two criteria: $\left|F_{l 1}^{2}\right| \rightarrow$ Min and $\left|v_{\text {ref } 0}-\dot{\xi}_{0}\right| \rightarrow$ Min. The simplex algorithm is used.

- The optimization of the speed of the leader vehicle: $\sum_{j=1}^{m}\left|\lambda_{j}-\dot{\xi}_{0, j}\right|^{2} \rightarrow$ Min. A simple matrix manipulation is used.

- The required longitudinal force is based on the robust control method. The robust control is designed in an off-line way.

\section{Design of vehicle control in the platoon}

\subsection{Design of robust control}

This section focuses on the design of the control input, i.e., the design of the longitudinal forces of the vehicles in the platoon. In the previous section the required reference speed of the leader vehicle was determined by taking the road inclinations into consideration. The speed of the leader vehicle must track the required speed. At the same time the other vehicles in the platoon must meet the string-stable requirement in order to guarantee the safe operation of the platoon. Consequently, two types of controllers must be designed: a speed tracking controller for the leader vehicle and a string stable controller for each vehicle in the platoon. All the controllers must provide disturbance attenuation and robustness against uncertainties.

The design of controllers are based on robust $\mathcal{H}_{\infty}$ methods. The control design of the leader vehicle is a tracking problem formulated in (1). The aim of tracking is to ensure that the system output follows a reference value of speed with an acceptable error, which is the performance of the system. The explicit mathematical description of the optimization problem is $\bar{\lambda}_{1}-\dot{\xi}_{0,1} \longrightarrow$ Min!, where the parameter $\bar{\lambda}_{1}$ is the required reference speed. The performance of the leader vehicle is as follows:

$$
z_{1}=\left[\bar{\lambda}_{1}-\dot{\xi}_{0,1}\right]
$$


The other vehicles in the platoon must guarantee string stability. This property of the system ensures that the inter-vehicular spacing errors of all the vehicles remain bounded uniformly in time, provided the initial spacing errors of all the vehicles are bounded. There are several strategies by which it is possible to ensure the string stability of the system. In the paper string stability is ensured by tracking the position, speed and acceleration of the preceding vehicle, and tracking the position and speed of the leader vehicle. [26] proves the applicability of this platoon model for control design.. The performance vector of the $j^{\text {th }}$ vehicle is:

$$
z_{j}=\left[\ddot{\varepsilon}_{j} ; \quad \dot{\varepsilon}_{j} ; \quad \varepsilon_{j} ; \dot{\xi}_{0, j}-\dot{\xi}_{0,1} ; \xi_{0, j}-\xi_{0,1}+\sum_{i=1}^{j} L_{i}\right]^{T}
$$

where $L_{i}$ is the distance between two vehicles and $\varepsilon_{i}=\xi_{0, j}-\xi_{0, j-1}$ is the error of tracking. Communication between the vehicles in the platoon is a crucial point of platoon control. The suitable intervehicle communication networks and the effects of communication delays to string stability are found in [14, 28].

The closed-loop interconnection structure, which includes the feedback structure of the model $P$ and controller $K$, is shown in Figure 4. The control design is based on a weighting strategy. The purpose of weighting function $W_{p}$ is to define the performance specifications of the control system, i.e., the speed of the vehicle must ensure the tracking of the reference signal with an acceptable error. The purpose of the weighting function $W_{n}$ is to reflect the sensor noise, while $W_{w}$ represents the effect of longitudinal disturbances. In the modeling an unstructured uncertainty is modeled by connecting an unknown but bounded perturbation block $(\Delta)$ to the plant. The magnitude of output multiplicative uncertainty is handled by the weighting function $W_{u}$.

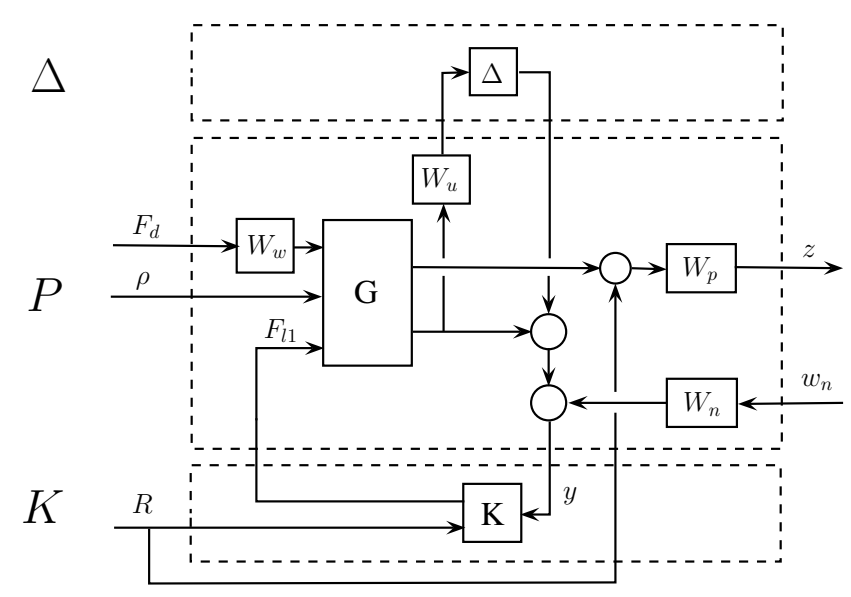

Figure 4: Closed-loop interconnection structure of the system

The performance signal $z$ differs in the two cases. In case of the leader $z=z_{1}$ according to (12) and in the other vehicles $z=z_{j}$ according to (13). For the leader vehicle the measured signals are the speed and the acceleration of the vehicle, while the follower vehicles also require the speed, position and acceleration information about the preceding and leader vehicles. The reference signal $R$ is the required reference speed $\bar{\lambda}_{1}$ at the leader vehicle, while for the follower vehicles $R$ vector contains the speed and position of the leader and the preceding vehicles and the acceleration of the preceding one. 


\subsection{Stability analysis of the closed-loop system}

The computation of $\lambda$ depends on speed $\dot{\xi}_{0}$ and acceleration $\ddot{\xi}_{0}$ of the vehicle and exogenous signals. The speed of the vehicle is considered at the computation of the reference speed signal, which is a feedback in the closed-loop system. The result of reference signal computation influences the stability of the controlled system.

Since the relationship between the vehicle parameters and $\lambda$ is rather complex, a simplification is used in the analysis. In the following a transfer function $N_{j}$ is introduced. It represents the relationship between momentary speed $\dot{\xi}_{0, j}$ and its reference $\lambda_{j}$. This model with an autoregressive structure (ARX) is identified, see [15]:

$$
y_{j}(q)=\frac{B_{j}(q)}{A_{j}(q)} u_{j}(q)+e(q)
$$

where $B(q) / A(q)$ is the discrete-time model of the continuous system $N_{j}, y(q)$ and $u(q)$ are discrete signals, which are sampled from $\lambda_{j}(t)$ and $\dot{\xi}_{0, j}(t)$, while $e(q)$ is white noise. According to $(7)$, it is possible to formulate a SIMO system with input $\bar{\lambda}_{1}$ and outputs $\dot{\xi}_{0, j}, j \in[1, n]$ :

$$
G_{p l}=\left[\begin{array}{llll}
\hat{G}_{1} & \hat{G}_{2} & \ldots & \hat{G}_{n}
\end{array}\right]^{T}
$$

The illustration of the controlled systems is shown in Figure 5. Then (11) is rearranged in a matrix form as:

$$
\bar{\lambda}_{1}=\frac{\sum_{j=1}^{n}\left(\lambda_{j} \prod_{k=1}^{j-1} \hat{G}_{k}\right)}{\sum_{j=1}^{n}\left(\prod_{k=1}^{j-1} \hat{G}_{k}\right)^{2}}=G_{\lambda} \xi
$$

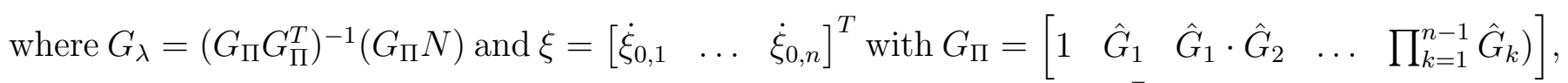
and $N=\operatorname{diag}\left(N_{1} ; N_{2} \ldots N_{n}\right) . G_{\text {filter }}$ is a low-pass filter, which smoothes $\bar{\lambda}_{1}$ reference signal. Note this function must be used because the necessary measurements for the computation of $\bar{\lambda}_{1}$ are sampled. $G_{\text {filter }}$ is also constant.

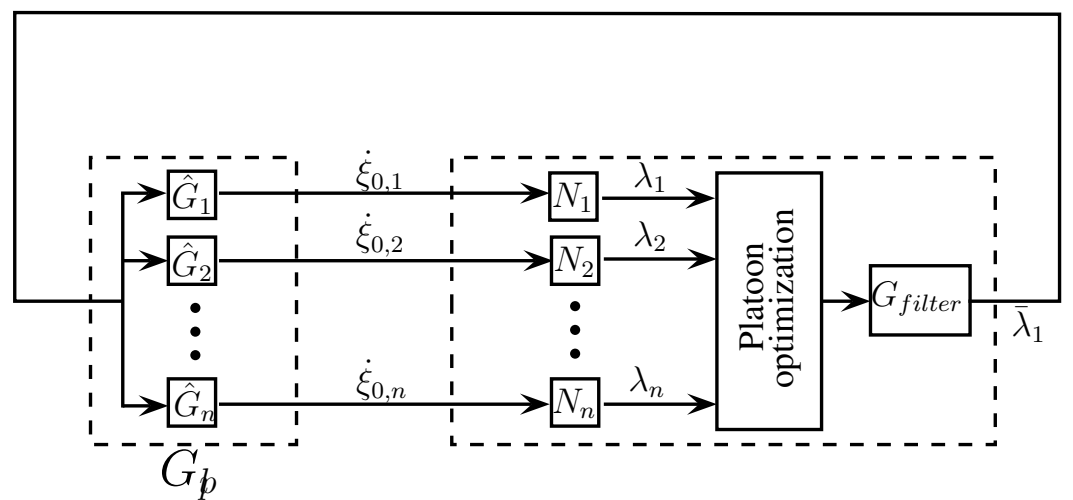

Figure 5: Closed-loop system of platoon control

The stability of the closed-loop system can be analyzed by the Small gain theorem [29]:

$$
\left\|G_{f i l t e r} G_{\lambda}\right\|\left\|G_{p l}\right\|<1
$$


(17) shows that the stability of the closed-loop system depends on the infinite norms of $G_{p l}, G_{\lambda}$, $G_{\text {filter }}$. Since these factors depend on dynamics and controllers of vehicles, infinite norm (17) depends on $N$, which is identified, see (14).

If (17) is not guaranteed by $\bar{\lambda}_{1}(k)$, then it is overwritten by $\hat{\lambda}_{1}(k-1)$, which is computed in step $k-1$. It means the reference signal is constant. Note this analysis is relevant mainly in practice, when $\lambda>\dot{\xi}_{0}$, i.e., the vehicle is accelerating, e.g., before an uphill section or when a speed limit is lifted.

\subsection{Implementation of the controller}

In the paper, the purpose of the control design is to calculate the necessary longitudinal control force. The paper focuses on this high-level controller $K$, which calculates positive and negative forces as well, therefore the traction and braking system are also actuated. It is assumed that there is another controller which is able to track the control force as a required force. This low-level Electronic Control Unit (ECU) transforms the longitudinal force into a real physical parameter of the actuator. The design of this low-level ECU may use more specific techniques that fit the specific nonlinear properties of the actuator. Figure 6 shows the architecture of the low-level ECU used in each vehicle.

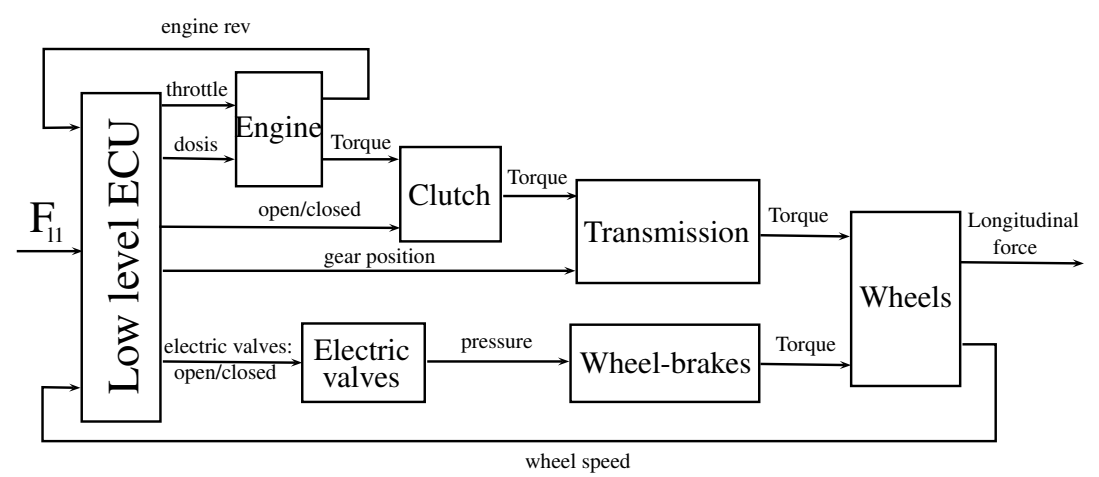

Figure 6: Architecture of the low-level controller

The engine-management system and fuel-injection system have their own controllers, thus in the realization of the low-level controller only the torque-rev-load characteristics of the engine are necessary. In this case the rev of the engine is measured, the required torque is computed from the longitudinal force of the high-level controller, thus the throttle is determined by an interpolation step using a look-up table. The position of the transmission is determined by logic functions, thus it depends on the fuel consumption and the maximal rev of the engine. The pressures on the brake cylinders of the wheels increase in case of braking. The necessary braking pressure for the required braking force is computed from the ratios of the hydraulic/pneumatic parts.

\section{Simulation results}

In this section a transportational route with real data is analyzed. The terrain characteristics and geographical information are those of the $A 8$ German motorway between Ulm and Stuttgart in a $90 \mathrm{~km}$ long section. The motorway $A 8$ runs across the Alps in South-Germany and connects 
Luxembourg with Austria, therefore it is one of the most important East-West transit routes. The chosen road section, especially the Swabian part contains uphill sections, in which the energy consumption of the platoon is critical. Publicly accessible up-to-date geographical/navigational databases and visualization programs, such as Google Earth and Google Maps, are used for the experiment. The platoon in the simulation contains four members, each of which is a $3500 \mathrm{~kg}$ light commercial vehicle (LCV). The recommended maximum speed on the motorway is $130 \mathrm{~km} / \mathrm{h}$ but the road section also contains speed limits (e.g. $50 \mathrm{~km} / \mathrm{h}$ or $80 \mathrm{~km} / \mathrm{h}$ ). The length of the predicted road horizon is $500 \mathrm{~m}$, which is divided into 10 points.

Figure 7(a) shows the altitude of the road section along the way. In this simulation two different controllers are compared. Controller 1 is a conventional platoon controller, which ignores

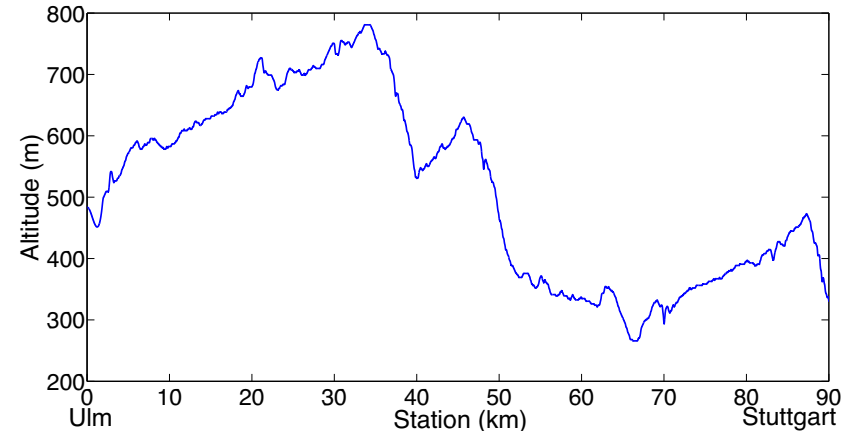

(a) Altitude

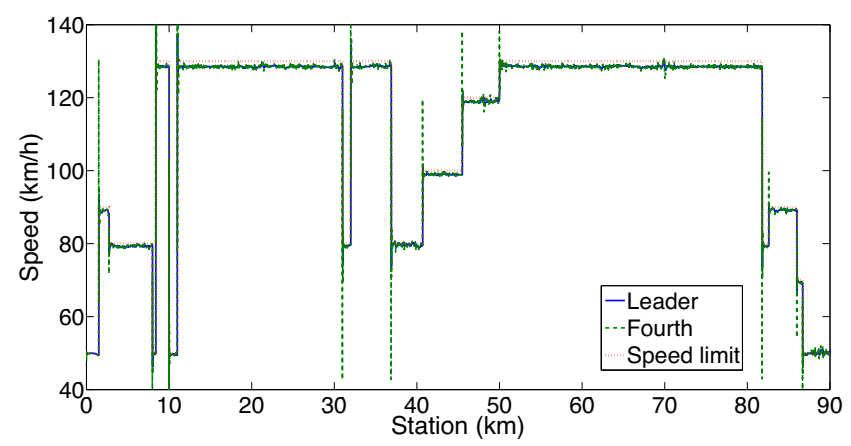

(c) Speed using Controller 1

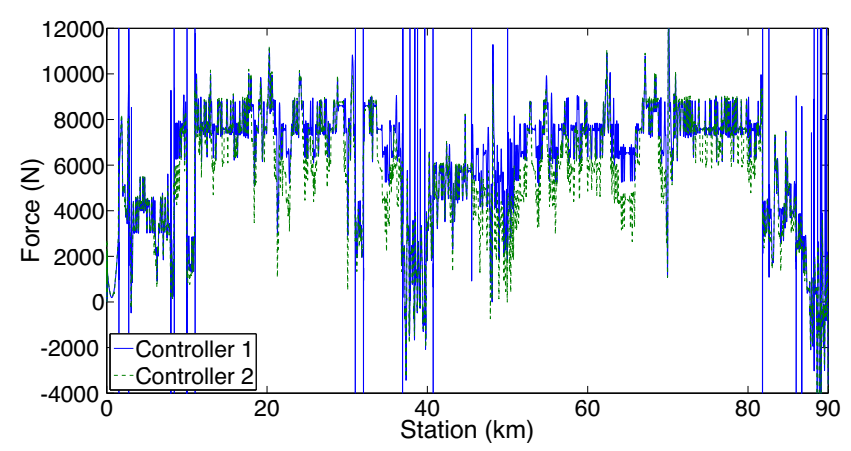

(e) Longitudinal force of the leader

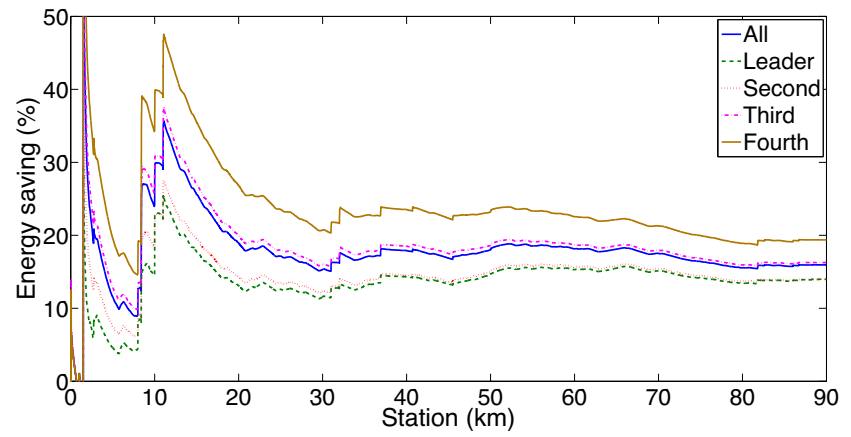

(b) Energy saving of the platoon

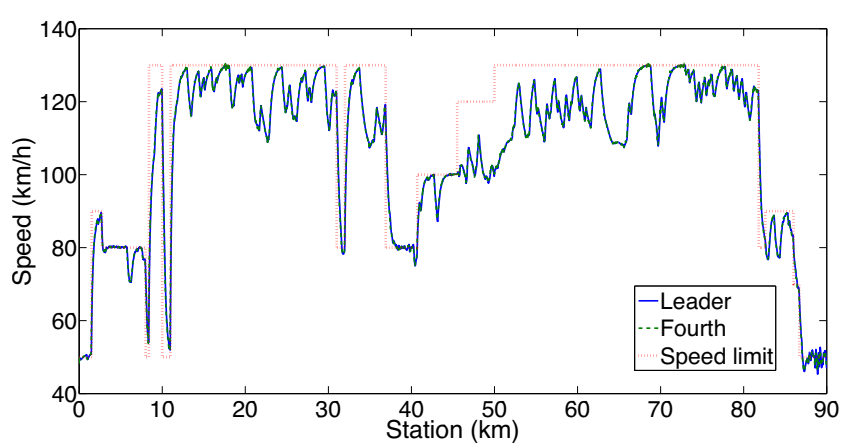

(d) Speed using Controller 2

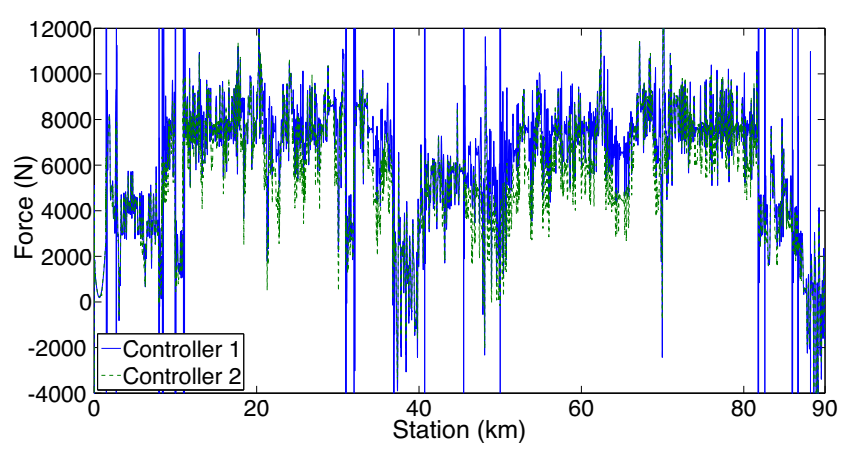

(f) Longitudinal force of the fourth vehicle

Figure 7: Real data motorway simulation

the road information, while Controller 2 is the proposed platoon controller, which considers the road conditions such as the inclinations and speed limits. Figure 7(c) illustrates the speed values of leader and the fourth vehicle of the platoon using Controller 1. It tracks the predefined speed 
limits as accurately as possible and the tracking error is minimal. Controller 2 modifies the speed values considering speed limits and road inclinations simultaneously according to the optimal requirement, see Figure $7(\mathrm{~d})$. For example, at $31 \mathrm{~km}$ of the road there is a speed limit, therefore the vehicles must be decelerated to $80 \mathrm{~km} / \mathrm{h}$. Controller 1 reduces the speed abruptly, while Controller 2 reduces the speed within a longer distance exploiting the adhesion of the road. In another example at $34 \mathrm{~km}$ of the road there is a downhill section after an uphill one. Controller 2 reduces the speed before the top of the section, because the vehicles are going to accelerate on the downhill slope. The longitudinal control forces of the leader and the fourth vehicle are presented in Figure 7(e) and Figure 7(f). The high-precision tracking of the predefined speeds in the conventional platoon control system often requires extremely high forces with abrupt changes in the signals. Since the proposed method calculates the longitudinal forces in advance based on the road inclinations and speed limits the truck is able to travel along the road with smaller actuation.

As a result of the road conditions less energy is required during the journey in the proposed control method, see Figure 7(b). It is shown that the saved energies are different at the platoon, in the case of leader it is $14 \%$, while in the fourth vehicle it is almost $20 \%$. The overall energy saving of the platoon is $16 \%$, which can be divided into brake actuation saving (88\%) and powertrain actuation saving (12\%). Fuel consumption can also be evaluated using the following simple equation: $V=E /\left(\eta L_{h} \rho_{\text {fuel }}\right)$, where $E$ is control energy, $\eta$ is the efficiency of the powertrain system, $L_{h}$ is heating value and $\rho_{\text {fuel }}$ is the density of gasoline. This approximation results in $5.9 l$ reduction in fuel consumption in the $90 \mathrm{~km}$ length section. Since in the method the speed of the vehicle may be below the permitted maximum for the given section and accelerations/decelerations are carried out more slowly and gradually than in the conventional method the duration of the journey is expected to be longer. However, this difference is only 2 minutes. Therefore the increase in travel time is $3.9 \%$.

The stability of the platoon system is analyzed using equation (17). Figure 8 shows the calculated norm of the closed platoon system. The value of the norm is below the upper bound 1, which guarantees the stability of the system by exploiting the Small gain theorem.

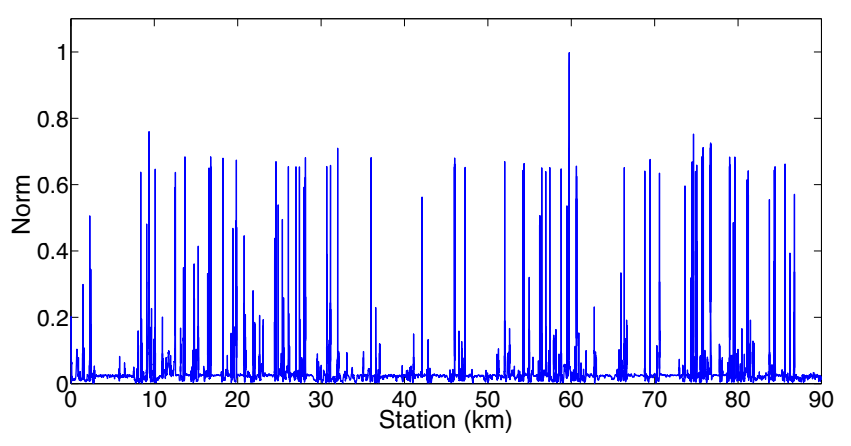

Figure 8: Stability norm of the platoon

\section{Conclusion}

The paper has proposed a control design method for a platoon system. The controlled system incorporates the brake and the traction forces. The method takes into consideration safe travel 
by using the string stability theorem and the knowledge of the inclinations of the road along the route of the platoon and the compulsory speed limits. By choosing the speed of the platoon fitting in with the inclinations of the road the number of unnecessary accelerations and brakings, i.e., the activations of the traction and brake systems, can be reduced. An $\mathcal{H}_{\infty}$-based control design is proposed for the leader and the members of the platoon. The controller is able to perform speed tracking, minimizes the actuator forces, thus reduces fuel consumption. The analysis of the string stability of the platoon is also performed in the control design. In a real data simulation example it is shown that the proposed method is able to save energy compared to conventional platoon systems.

\section{Acknowledgements}

The research was supported by the Hungarian National Office for Research and Technology through the project "Innovation of distributed driver assistance systems for a commercial vehicles platform" (TECH_08_2/2-2008-0088).

\section{References}

[1] H. S. Bae, J. Ruy, and J. Gerdes. Road grade and vehicle parameter estimation for longitudinal control using gps. 4th IEEE Conference on Intelligent Transportation Systems, 2001.

[2] M. Barth, F. An, J. Norbeck, and M. Ross. Modal emissions modeling: A physical approach. Transportation Research Record, (1520):81-88, 1996.

[3] T. F. Coleman and Y. Li. A reflective newton method for minimizing a quadratic function subject to bounds on some of the variables. SIAM Journal on Optimization, 6(4):1040-1058, 1996.

[4] S.J. Deutschle, G.C. Kessler, M. Hakenberg, and D. Abel. The konvoi project: Development and investigation of truck plaoons on highways. FISITA Congress, Budapest, 2010.

[5] W.F. Faris, H.A. Rakha, M. Idres R. I. Kafafy, and S. Elmoselhy. Vehicle fuel consumption and emission modelling: an in-depth literature review. International Journal of Vehicle Systems Modelling and Testing, 6(3):318-395, 2011.

[6] P. E. Gill, W. Murray, and M.H. Wright. Practical Optimization. Academic Press, London UK, 1981.

[7] J.O. Hahn, R. Rajamani, S.H. You, and K.I. Lee. Real-time identification of road-bank angle using differential GPS. IEEE Transactions on Control Systems Technology, 12:589-599, 2004.

[8] H. Hanabusa, T. Shiraishi, K. Koide, R. Horiguchi, S. Tanaka, M. Kuwahara, T. Oguchi, and H. Oneyama. Development of a hybrid traffic simulation framework for environmental evaluation for its applications. $18^{\text {th }}$ World Congress on Intelligent Transport Systems, Orlando, USA, 2011.

[9] E. Hellström, M. Ivarsson, J. Åslund, and L. Nielsen. Look-ahead control for heavy trucks to minimize trip time and fuel consumption. Control Engineering Practice, 17(2):245-254, 2009.

[10] R. Horiguchi, H. Hanabusa, M. Kuwahara, S. Tanaka, T. Oguchi, H. Oneyama, H. Hirai, and S. Hayashi. Validation scheme for traffic simulation to estimate environmental impacts in 'EnergyITS Project'. $17^{\text {th }}$ ITS World Congress, Busan, 2010. 
[11] M. Ivarsson, J. Åslund, and L. Nielsen. Look ahead control - consequences of a non-linear fuel map on truck fuel consumption. Proc. Institution of Mechanical Engineers, Part D, J. Automobile Engineering, 223:1223-1238, 2009.

[12] R. Labayrade, D. Aubert, and J.P. Tarel. Real time obstacle detection in stereovision on non flat road geometry through "v-disparity" representation. Intelligent Vehicle Symposium IEEE, 2:646$651,2002$.

[13] M. Larburu and J. Sanchez. Safe road trains for environment: Human factors' aspects in dual mode transport systems. 17th World Congress on Intelligent Transport Systems, Busan, Korea, 2010.

[14] X. Liu, A. Goldsmith, S.S. Mahal, and J.K. Hedrick. Effects of communication delay on string stability in vehicle platoons. IEEE Proceedings of Intelligent Transportation Systems, pages 625$630,2001$.

[15] L. Ljung. System Identification: Theory for the User. Prentice Hall, Upper Saddle River, 1999.

[16] D.W. Marquardt. An algorithm for least-squares estimation of nonlinear parameters. Journal of the Society for Industrial and Applied Mathematics, 11(2):431-441, 1963.

[17] L. Nouveliere, M. Braci, L. Menhour, and H.T. Luu. Fuel consumption optimization for a city bus. UKACC Control Conference, Manchester, 2008.

[18] B. Németh and P. Gáspár. LPV-based control design of vehicle platoon considering road inclinations. IFAC World Congress, 2011.

[19] B. Passenberg, P. Kock, and O. Stursberg. Combined time and fuel optimal driving of trucks based on a hybrid model. European Control Conference, Budapest, 2009.

[20] H.A. Rakha, A. Kyoungho, W. Faris, and K.S. Moran. Simple vehicle powertrain model for modeling intelligent vehicle applications. IEEE Transactions on Intelligent Transportation Systems, 13(2):770780,2012 .

[21] G. Rödönyi, P. Gáspár, J. Bokor, Sz. Aradi, Z. Hankovszki, and L. Palkovics R. Kovács. Guaranteed peaks of spacing errors in an experimental vehicle string. 17th IFAC Symposium on Robust Control Design, Aalbord, Denmark, 2012.

[22] B. Saerens, H.A. Rakha, M. Diehl, and E. Van den Bulck. A methodology for assessing eco-cruise control for passenger vehicles. Transportation Research Part D, 19:20-27, 2013.

[23] E. Shaw and J.K. Hedrick. String stability analysis for heterogeneous vehicle strings. American Control Conference, pages 3118 - 3125, 2007.

[24] S. Sheikholeslam and C. A. Desoer. Longitudinal control of a platoon of vehicles. III, nonlinear model. Research Report. Institute of Transportation Studies, UC Berkeley, 1990.

[25] Z. Sitavancova and M. Hajek. Promote-chauffeur: Intelligent transport systems. EU Mobility and Transport, 2010.

[26] D. Swaroop and J.K. Hedrick. String stability of interconnected systems. IEEE Transactions on Automatic Control, 41:349-357, 1996.

[27] Project TruckDAS/Platooning. Innovation of distributed driver assistance systems for commercial vehicle platform, November 2011. http://www.truckdas.hu/. 
[28] T. H. Willke, P. Tientrakool, and N. F. Maxemchuk. A survey of inter-vehicle communication protocols and their applications. IEEE Communications Surveys \& Tutorials, 11(2), 2009.

[29] K. Zhou, J.C. Doyle, and K. Glover. Robust and Optimal Control. Prentice Hall, 1996.

[30] W. Zhu and M. Barth. Vehicle trajectory-based road type and congestion recognition using wavelet analysis. IEEE Intelligent Transportation Systems Conference, Toronto, Canada, pages 879-884, 2006. 